general. It does not seem impossible that it is just this property which determines the difference in the organization of plants and lower animals on one hand and of the warm-blooded animals on the other: We may in this connexion recall, for example, the great capacity for regeneration of plants and lower animals as compared with higher animals, and the occurrence of both sex and other mosaics in plants and insects and their absence in warm-blooded animals.

Our thanks are due to Prof. A. Turunen, head of the Women's Clinic of the University of Helsinki, for the opportunity of carrying out the present study. A more extensive account of these observations and their theoretical implications will be published elsewhere ${ }^{10}$.

Women's Clinic,

University of Helsinki.

$$
\text { Aug. } 15 .
$$

${ }^{1}$ Geitler, L., "Chromosomenbau" (Berlin, 1938).

${ }^{2}$ Oksala, T., Hereditas, 25, 132 (1939).

${ }^{3}$ Huskins, C. L., Proc. 8th Internat. Congress Genetics, 274 (1948).

- Karplus, H., Z. Zellf., 10, 38 (1930).

Andres, A. H., and Jiv, B. V., Cytologia, 7, 371 (1936).

- Kemp, T., Z. Zellf., 11, 429 (1930).

7 Timonen, S., and Therman, E., Cancer Research, 10, 431 (1950).

${ }^{s}$ Therman, E., and Timonen, S., Bereditas (in the press).

- Timonen, S., Acta Obstetr. Scand., 31, Supp. 2, 1 (1951).

${ }^{10}$ Therman, E., and Timonen, S., Hereditas (in the press).

\section{Multiple Sex Chromosomes in the Marsupials}

DURING the past thirty years, some twenty species of marsupials have been examined cytologically. In all of them, a typical $X Y$ sex-chromosome mechanism has been reported. We have recently re-investigated the potoroo (Potorous tridactylus), a common species of rat-kangaroo in Tasmania. In the spermatogonial mitoses, the male shows thirteen chromosomes, whereas the female shows twelve in ovarian mitoses (Figs. 1 and 2). The chromosomes of the female can easily be arranged in homologous pairs, one pair (the $X$-chromosomes) heing conspicuous by their long, nearly median, centromere constriction. In the male, ten chromosomes can be paired, but three have no similar partners. These are the $X$, with a long submedian constriction, the smallest of the set $\left(Y_{1}\right)$, and an acrocentric $\left(Y_{2}\right)$. The last two chromosomes are
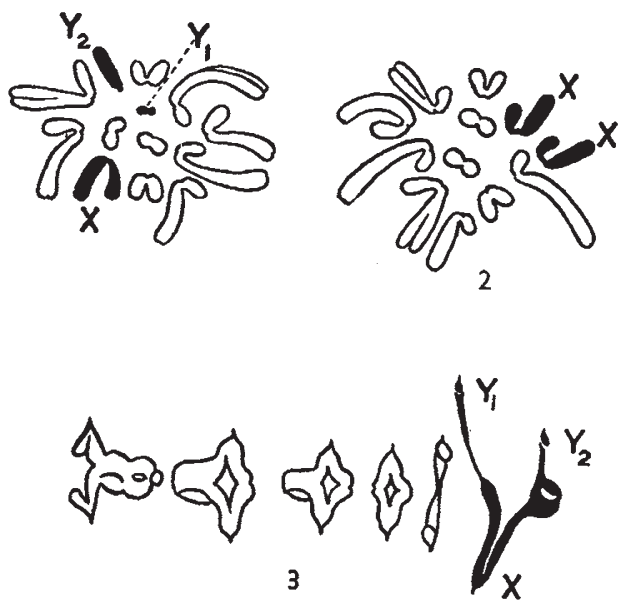

Magniflcatlon, 2,000 not represented in the female. In accord with these observations, five bivalents held together by from one to five chiasmata and a trivalent are formed at the first division of meiosis in the male (Fig. 3). The trivalent is made up of the $X$, pairing in one of its arms with $Y_{1}$, and in the other arm with $Y_{2}$. Orientation on the spindle is always such that $Y_{1}$ and $Y_{2}$ are directed to one pole, and the $X$ to the other pole. Two types of sperm are produced, a maledetermining type with seven chromosomes, and a female-determining with six. The sex-determining mechanism is, thus, of the $X Y_{1} Y_{2}: X X$ type.

In the prophase stages of meiosis, the $Y_{2}$ and its homologous arm in the $X$ behave similarly to the autosomes and usually form two typical chiasmata. The other arm of the $X$-chromosome, like $Y_{1}$, is strongly heterochromatic, being condensed into an irregular sphere at earliest diplotene. At later diplotene this arm is seen to be made up of two parts, the distal pairing segment being more condensed than the intercalary unpaired region. This behaviour is characteristic of the $X$-chromosomes of other marsupials that have been investigated ${ }^{1}$. It is thus probable that the aberrant mechanism in the potoroo has arisen by translocation between the $X$-chromosome and an autosome with loss of a centromere.

These results are based on the examination of three specimens, a male from Sorell in southern Tasmania, and a male and female obtained in Hobart. The species has previously been investigated by Altmann and Ellery ${ }^{2}$ in animals obtained from Tasmania. They interpreted their observations as showing a typical $X Y$ mechanism. It is, perhaps, possible that two sex-determination mechanisms exist in this species. However, since their $X Y$ bivalent corresponds in size to the smallest bivalent of our material, this seems unlikely.

It is at present impossible to say how widespread multiple sex-chromosome mechanisms are in the marsupials. As pointed out by Darlington ${ }^{8}$ and more recently by Matthey ${ }^{4}$, Agar's ${ }^{5}$ results on Macropus ualabatus (= Wallabia bicolor $)$ are probably to be interpreted as showing the existence of a multiple mechanism in this species. Indeed, the $X$-chromosome seems to have the same long centromere constriction which is visible in the potoroo. We interpret Agar's figures as showing that the female wallaby has ten chromosomes; and the male eleven, of which three form a trivalent at meiosis as in the potoroo. It is probable that a similar mechanism may also exist in the insectivore Sorex araneus, Bovey (cited in Matthey ${ }^{4}$ having described a trivalent at meiosis in the male. The chromosome number in the female of this species is, however, unknown.

We wish to thank Mr. A. G. Lyne for assistance in this investigation. The Fauna Board of Tasmania kindly gave us permission to take specimens of this protected animal. One of us was in receipt of a Commonwealth Research Scholarship.

G. B. SHARMAN

A. J. McIntosh

Department of Botany, H. N. BARBER

University of Tasmania. Aug. 8.

${ }^{1}$ Koller, P. C., J. Genet., 32, 451 (1936).

"Altmann, S. C., and Ellery, M. E., Quart. J. Micr. Sci., 69, 463 (1925).

- Darlington, C. D. " "Recent Advances in Cytology" (London, 1937).

"Matthey, R., "Les Chromosomes des Vertébrés" (Lausanne, 1949).

- Agar, W. E., Quart. J. Micr. Sci., 67, 183 (1923). 